\title{
Response to Liu et al.
} Mark O. Goodarzi, MD, $\mathrm{PhD}^{1}$ and
David C. Whitcomb, MD, $\mathrm{PhD}^{2,3,4}$

Clinical and Translational Gastroenterology 2019;10: e00096. https://doi.org/10.14309/ctg.0000000000000096

We thank Liu et al. for their letter and hope that the response will answer their concerns and will also be informative to others who are confused about these issues. Three points will be addressed.

\section{RELATIONSHIP BETWEEN \\ RECURRENT ACUTE \\ PANCREATITIS AND \\ CHRONIC PANCREATITIS}

The clinicopathologic definitions of recurrent acute pancreatitis (RAP) and chronic pancreatitis (CP) are based on distinguishing clinical features of complex syndromes (e.g., $>1$ episode of AP for the diagnosis of RAP and morphologic features of fibrosis for the diagnosis of CP) with no reference to etiology. In the 1960 s, attempts were made to distinguish RAP from CP ((1), see also Etemad and Whitcomb (2)), but the discovery that gain-of-function mutations p.R122H or p.N29I in the cationic trypsinogen gene (PRSS1) cause AP and RAP (primary phenotype), with a subset later developing $\mathrm{CP}$, clarified the progressive relationship between these clinical syndromes (3).

The etiology-based, progressive relationship between RAP and CP is specifically defined in the Mechanistic Definition of CP (4), a new definition that has been accepted by the major pancreatic societies (5). Multiple studies, including the meta-analysis (6) noted by Liu et al., confirm the relationship between RAP and $\mathrm{CP}$, but they do not infer that all RAP becomes CP nor that $\mathrm{CP}$ requires RAP. Note also that the diagnosis of RAP does not preclude the diagnosis of $\mathrm{CP}$ or vice versa, and they can occur in the same person at the same time.

The evidence that Liu et al. use to argue against including RAP with $\mathrm{CP}$ in our analysis is based on an article by Cavestro et al. (7). However, this article is now a decade out of date, grossly underpowered (RAP $n=64$; CP $n=142$ ), and only looked at 1 variant in $M C P-1,1$ in SPINK1, and 33 cystic fibrosis-causing variants in CFTR. Their limited data suggested that genetic variants cause RAP but not CP. More recent studies that are larger and/or that have deeper genotyping demonstrate that RAP and CP have common etiologies and that time and persistent injury or inflammation, or additional risk factors that are present within a subset of RAP patients, cause progression to $\mathrm{CP}$ (8-12). Finally, the etiology of diabetes mellitus (DM) is linked to the pancreatic beta cell, not the acinar or duct cells that contribute to the etiology of RAP and CP. The fact that even one episode of AP clearly increases risk of DM (13) suggests that in some patients, susceptibility to DM is clearly linked to exocrine pancreatic injury and inflammation-which takes the forms of AP, RAP, and CP.

\section{NORTH AMERICAN PANCREATITIS STUDY II}

We are happy to clarify the study cohorts used by Goodarzi et al. (14) related to the North American Pancreatitis Study II (NAPS2) samples. Liu et al. inferred bias in sample selection of this study based on counting sample from 2 previous reports using NAPS2 samples $(9,15)$. However, a simple reading of the methods papers reveals that the genome-wide association study included only cases (both RAP and $\mathrm{CP}$ ) and controls of European ancestry and additional non-NAPS2 samples from England, Germany, and the United States. This is appropriate for detecting genetic variants associated with pancreatitis. However, the non-NAPS2 samples were not phenotyped for DM and could not be used in more detailed analyses.

The second article referenced by Liu et al. focused on patients with non-European ancestry (i.e., African Americans) (15) and did not include the genotyping results, since the initial genome-wide association study chip was designed for patients of European ancestry (9). Furthermore, there was continued patient recruitment to the Pittsburgh site, further increasing the overall cohort size.
The sample selected for the study by Goodarzi et al. (14) includes only NAPS2 subjects since they had detailed information of the presence of absence of $\mathrm{DM}$, the age of DM and AP, RAP and CP onset, and medications. All NAPS2 subjects with complete case report forms, and in which genotyping on all SNPs were successfully genotyped, were indeed included.

Liu et al. also raised concerns about a selection bias for not having follow-up data on the patients for at least 2-5 years to exclude pancreatic cancer, referencing Kirkegard et al. (16). We are unclear as to the relevance of this criticism since (i) the study by Kirkegard focused on AP, not CP, (ii) the follow-up time requirement was to insure that AP was not caused by pancreatic cancer, (iii) the study used an administrative data set, so that unlike the NAPS2 patients, the cases were not phenotyped by an expert physician with access to imaging studies and laboratory values within a clinical context, and (iv) even after 5 years of follow-up, the incidence of pancreatitis cancer was less than $1 \%$. Although it is also possible that patients with CP may develop pancreatic cancer and that a subset of these patients may have glucose intolerance or DM (17), the mechanism is likely paraneoplastic and would bias the study against type 2 DM (T2DM) genetics (18). Thus, we do not believe the study by Goodarzi was biased by contamination by patients with pancreatic cancer.

\section{HETEROGENEITY OF \\ DIABETES ETIOLOGY}

Liu et al. suggest that we did not clearly highlight the heterogeneity within the group with CP and diabetes (CP-DM). On the contrary, we discussed at length the likelihood that the CP-DM group included people with diabetes caused by $\mathrm{CP}$ and people with classic T2DM. We even acknowledged that both etiologies may contribute to diabetes in some individuals. We explicitly stated that such heterogeneity could have reduced the ability for the genetic risk score (GRS) to distinguish the CP-DM group from the T2DM group. The potential heterogeneity is why we conducted additional analyses attempting to enrich 
the CP-DM group with pancreatogenic diabetes or deplete the group of T2DM. Regarding the latter, Liu et al. suggest we should have simultaneously excluded patients with multiple T2DM-related features in subanalysis. We had examined the strategy of stratifying on multiple features but found that it resulted in CP-DM subgroups of small size and generally did not add insight beyond considering single features. As an example, we provide the analysis requested by Liu et al. A CP-DM subgroup of 63 patients had diabetes occurring after pancreatitis, no family history of diabetes, and was not obese or overweight. Their mean GRS was 66.21, which was essentially identical to the mean GRS of 66.42 in the 423 patients with T2DM $(P=0.93)$.

Thus, we stand by the study design and the conclusions and hope that this additional explanation of our previous articles and review of the genetics literature will bring clarity to this changing field.

\section{CONFLICTS OF INTEREST}

Guarantor of the article: Mark O. Goodarzi, $\mathrm{MD}, \mathrm{PhD}$.

Specific author contributions: M.O.G., D.C.W.: drafting of manuscript.

Financial support: This research was partly supported by NIH grants R01-DK061451 (DCW), U01-DK108314 (MOG), U01DK108306 (DCW), P30-DK063491 (MOG), and the Eris M. Field Chair in Diabetes Research (MOG). The study sponsors had no role in the study design, collection, analysis or interpretation of data, in the writing of the report, or in the decision to submit the report for publication.

Potential competing interests: D.C.W. is a consultant for AbbVie, Regeneron, and Ariel Precision Medicine and has equity in Ariel Precision Medicine. M.O.G. has no conflicts to disclose.

\section{REFERENCES}

1. Sarles H. Proposal adopted unanimously by the participants of the Symposium, Marseilles 1963. Bibliotheca Gastroenterologica 1965;7: 7-8.

2. Etemad B, Whitcomb DC. Chronic pancreatitis: Diagnosis, classification, and new genetic developments. Gastroenterology 2001;120:682-707.

3. Gorry MC, Gabbaizedeh D, Furey W, et al. Mutations in the cationic trypsinogen gene are associated with recurrent acute and chronic pancreatitis. Gastroenterology 1997; 113:1063-8

4. Whitcomb DC, Frulloni L, Garg P, et al. Chronic pancreatitis: An International draft consensus proposal for a new mechanistic definition. Pancreatology 2016;16:218-24.

5. Whitcomb DC, Shimosegawa T, Chari ST, et al. International consensus statements on early chronic pancreatitis. Recommendations from the working group for the international consensus guidelines for chronic pancreatitis in collaboration with the International Association of Pancreatology, American Pancreatic Association, Japan Pancreas Society, PancreasFest Working Group and European Pancreatic Club. Pancreatology 2018.[Epub ahead of print May 21, 2018.]

6. Sankaran SJ, Xiao AY, Wu LM, et al. Frequency of progression from acute to chronic pancreatitis and risk factors: A metaanalysis. Gastroenterology 2015;149: 1490-500.

7. Cavestro GM, Zuppardo RA, Bertolini S, et al. Connections between genetics and clinical data: Role of MCP-1, CFTR, and SPINK-1 in the setting of acute, acute recurrent, and chronic pancreatitis. Am J Gastroenterol 2010;105:199-206.

8. Kumar S, Ooi CY, Werlin S, et al. Risk factors associated with pediatric acute recurrent and chronic pancreatitis: Lessons from INSPPIRE. JAMA Pediatr 2016;170:562-9.

9. Whitcomb DC, LaRusch J, Krasinskas AM, et al. Common genetic variants in the CLDN2 and PRSS1-PRSS2 loci alter risk for alcoholrelated and sporadic pancreatitis. Nat Genet 2012;44:1349-54.

10. Takeyama Y. Long-term prognosis of acute pancreatitis in Japan. Clin Gastroenterol Hepatol 2009;7:S15-7.

11. Weiss FU, Hesselbarth N, Parniczky A, et al. Common variants in the CLDN2-MORC4 and PRSS1-PRSS2 loci confer susceptibility to acute pancreatitis. Pancreatology 2018. [Epub ahead of print June 1,2018.]
12. Poddar U, Yachha SK, Borkar V, et al. Is acute recurrent pancreatitis in children a precursor of chronic pancreatitis? A long-term followup study of 93 cases. Dig Liver Dis 2017;49: 796-801.

13. Das SL, Singh PP, Phillips AR, et al. Newly diagnosed diabetes mellitus after acute pancreatitis: A systematic review and metaanalysis. Gut 2014;63:818-31

14. Goodarzi MO, Nagpal T, Greer P, et al. Genetic risk score in diabetes associated with chronic pancreatitis versus type 2 diabetes mellitus. Clin Transl Gastroenterol 2019;10: e00057.

15. Wilcox CM, Sandhu BS, Singh V, et al. Racial differences in the clinical profile, causes, and outcome of chronic pancreatitis. Am J Gastroenterol 2016;111:1488-96.

16. Kirkegard J, Cronin-Fenton D, HeideJorgensen U, et al. Acute pancreatitis and pancreatic cancer risk: A nationwide matched-cohort study in Denmark. Gastroenterology 2018;154:1729-36.

17. Chari ST, Leibson CL, Rabe KG, et al. Pancreatic cancer-associated diabetes mellitus: Prevalence and temporal association with diagnosis of cancer. Gastroenterology 2008;134:95-101.

18. Sagar G, Sah RP, Javeed N, et al. Pathogenesis of pancreatic cancer exosome-induced lipolysis in adipose tissue. Gut 2016;65: 1165-74.

${ }^{1}$ Division of Endocrinology, Diabetes, and Metabolism, Department of Medicine, CedarsSinai Medical Center, Los Angeles, California, USA; '2Division of Gastroenterology, Hepatology, and Nutrition, Department of Medicine, University of Pittsburgh and UPMC Medical Center, Pittsburgh, Pennsylvania, USA;

${ }^{3}$ Department of Human Genetics, Graduate School of Public Health, University of Pittsburgh, Pittsburgh, Pennsylvania, USA; ${ }^{4}$ Department of Cell Biology and Molecular Physiology, University of Pittsburgh, Pittsburgh, Pennsylvania, USA. Correspondence: Mark O. Goodarzi, MD, PhD. E-mail: mark.goodarzi@cshs.org.

(C) 2019 The Author(s). Published by Wolters Kluwer Health, Inc. on behalf of The American College of Gastroenterology

Open Access This is an open access article distributed under the Creative Commons Attribution License 4.0 (CCBY), which permits unrestricted use, distribution, and reproduction in any medium, provided the original work is properly cited. 